\title{
Cell-Culture Measurements Using Voltage Oscillations
}

\author{
A. Maldonado, G. Huertas, A. Rueda, J.L. Huertas \\ Instituto de Microelectrónica de Sevilla, \\ Dpt. Electronic and Electromagnetism, F. Physics, \\ Universidad de Sevilla, CSIC. \\ Sevilla, España.
}

\author{
P. Pérez, A. Yúfera \\ Instituto de Microelectrónica de Sevilla, \\ Dpt. Electronic Technology, ETSII, \\ Universidad de Sevilla, CSIC. \\ Sevilla, España.
}

\begin{abstract}
A comprehensive system for real-time monitoring of a set of cell-cultures using a Voltage Oscillation (VO) methodology is proposed. The main idea is to connect the bio-electrical elements (electrodes \& cell-culture) in such a way that sequentially a suitable electrical oscillator, which only uses a DC power source, is built. Using the employed electrical models given in $[1,2]$, the attained oscillation parameters (frequency and amplitude) can be directly related to the biological test. A digital circuitry is developed to pick-up the experimental measurements, which are gathered and shown in real-time in a web application.
\end{abstract}

Keywords- Bioimpedance, real-time monitoring, microelectrode, oscillation-based systems.

\section{INTRODUCTION}

Nowadays, many Lab-on-a-Chips (LoCs) and implanted devices for biomedical applications are based on sensing the impedance of a biological sample as one of the main biomarkers $[3,7,8,9,11,15]$. Among Impedance Spectroscopy (IS) techniques, Electrical Cell-substrate Impedance Spectroscopy (ECIS) was described $[4,5]$ as an useful alternative for Cell Culture (CC) assays, allowing real-time monitoring, and greatly reducing the cost and human effort in assays. Recently, an Oscillation-Based (OB) technique was proposed [10]. Data is obtained by measuring the frequency and amplitude in an oscillation circuit, on the basis of the electrical model knowledge of the system composed by two electrodes in series with the cells being measured.

In this work, it is proposed to reuse the circuits in [10] to study the state and time evolution of a set of cell-cultures in a real environment. To accomplish such purpose, several tasks must be completed; first of all, the electrical model of the system, the electrode\&cell-culture under study, must be properly defined [10]. Secondly, the OB circuits in [10] have to be tuned and improved for this particular application to match both the working frequency range and the amplitude of interest (the system sensitivity range of the oscillation parameters must be studied and analysed and the electrical signals must fulfil the level limitations for cell-cultures). It is proposed herein to obtain the parametric curves describing the bio-impedance evolution in time from the measurements of the frequency and the amplitude of the proposed circuit

This work was supported in part by the Spanish funded Project: TEC 2013-46242-C3-1-P: Integrated Microsystems for Cell Culture Assays, co-financed with FEDER. oscillator. Finally, a measurement system has been developed to acquire the experimental results.

Most impedance measurement approaches consider an "excitation" at a given frequency and measure the obtained response [3, 4]. Our work transforms the bio-impedance under test into an oscillator, adding some extra external components to force the "biological circuit" to oscillate. Those changes operated at the cell-culture under test will produce observable changes in the oscillation frequency/amplitude and subsequently, we can avoid any extra circuitry for ac stimuli generation and accurate impedance measurement.

To apply OV to bio-impedance measurements, a positive feedback loop is implemented. It is particularly important to predict accordingly the oscillation parameters (frequency and amplitude), either analytically or by the means provided by simulation software [13], as well as to avoid any dependencies of these parameters on the active element saturation levels. The proposed solution relies on a nonlinear element (comparator) located at the feedback loop to guarantee self-maintaining oscillations [14], and a Band-Pass (BP) filter in the loop, as shown in Fig.1. Under this configuration an oscillation condition is generated for each oscillation state. As a consequence, the main oscillation parameters are function of the bio-impedance, called herein Zx. This work presents an important improvement to other reported solutions [10].

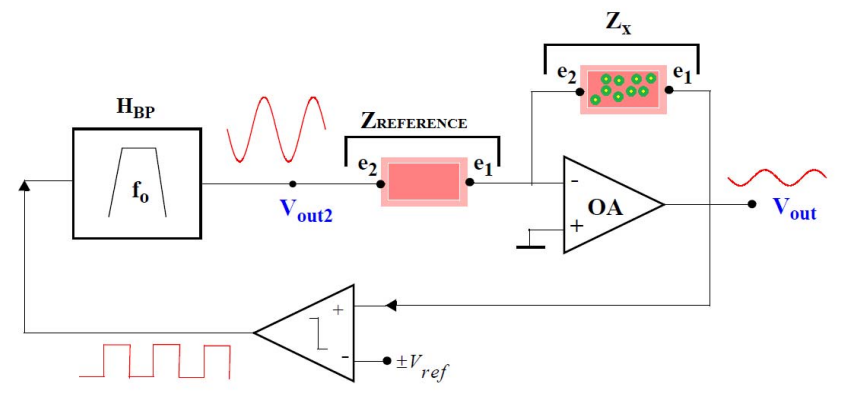

Fig. 1: OV block diagram. Proposed system set-up. A non-linear element, the comparator, and a second-order Band-Pass filter, HBP, with a central frequency, fo, are introduced at the positive feedback to force the system oscillations. To obtain suitable signal control, we employed an reference electrode at the inverting opamp configuration. 
The idea is to perform bio-impedance measurements by using an electrode of reference (cell empty, Zreference) to calibrate the bio-electrical system (formed by the bioimpedance under test connected in inverting configuration as shown in Fig. 1). This work analyzes the electrical model proposed for the micro-electrode\&cell-culture and the setup employed for measuring in section II. The system block diagram is described, mathematically analyzed and simulated in section III, demonstrating the correct values for the frequency and amplitude of oscillations obtained for the proposed test. In section IV, a description of the processing and the displaying part is shown. Final conclusions are summarized in section $\mathrm{V}$.

\section{ELECTRODE-CELL CULTURE ELECTRICAL MODEL}

How relevant is the Electrode\&Cell-Culture electrical model for testing bio-samples is directly related to the employed setup as described in [1-4]. It is proposed the measurement of a bioimpedance using two-wire setup. The electrodes are on the current signal path, and the voltage response measurement includes the cell-culture resistance plus the electrode response. Knowledge of cell-culture bioimpedance is required for adequate data extraction, it has been considered the model proposed in [1-4] for each electrode, in a two-wire setup (Fig. 2). For an electrode in saline solution, we have to consider the double-layer capacitance $(\mathrm{Cdl})$, the transfer resistance $(\mathrm{Rp})$, and the spreading resistance (Rs) for electrodes non-covered by cells. When any cell attachment happens, a new circuit element, i.e., the gap resistance (Rgap), models this effect [6] The model is valid only for ac voltages with amplitude below 100 $\mathrm{mV}[1,2]$. In this paper, the use of circular gold microelectrodes of $250-\mu \mathrm{m}$ diameter has been considered. Those electrodes can be totally or partially covered by cells in the culture. Henceforth, the fill factor (ff) parameter represents the percent of the electrode area (A) covered by cells (Ac). Values employed in the proposed analysis correspond to those given in [10].

In Fig. 3, it is displayed the electrode\&cell impedance Bode for different values of ff. Therefore, for a frequency of 11 $\mathrm{kHz}$ (peak frequency for the presented $\mathrm{BP}$ filter), an important change in the magnitude and the phase of the achieved Bode graphics is noticed. Observing the curves in Fig. 3 and extracting the appropriate information, it is proven that significantly different values of magnitudes and phases in the working frequency for each particular ff are achieved (Table I).

\begin{tabular}{|c|c|c|}
\hline \multicolumn{3}{|c|}{ Inverter Amplifier Gain (11 kHz) } \\
\hline ff & Magnitude $(\mathrm{dB})$ & Phase $(\mathrm{deg})$ \\
\hline $\mathbf{1 0} \%$ & 0.00 & 0.01 \\
$\mathbf{3 0 \%}$ & 0.731 & 7.27 \\
$\mathbf{5 0 \%}$ & 2.20 & 16.1 \\
$\mathbf{7 0 \%}$ & 4.17 & 27.7 \\
$\mathbf{1 0 0 \%}$ & 7.04 & 54.9 \\
\hline
\end{tabular}

Table I: Magnitude and phase values of inv. amplifiers in Fig. 1.

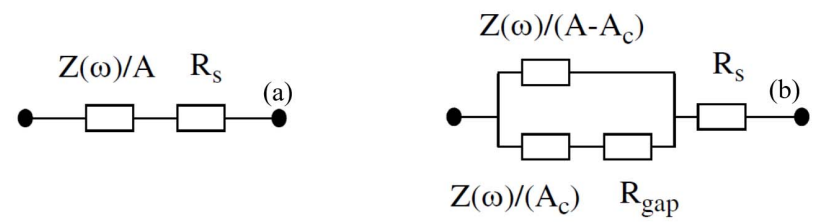

Fig. 2: Elements for the electrode-cell electrical model: (a) a bare electrode of area A and (b) an electrode of area A, partially covered with cells, an area $A_{c} \cdot Z(\omega)$ is the parallel connection between a capacitor and a resistor whose values are extracted from $[1,2] . \mathrm{R}_{\mathrm{s}}=5.4 \mathrm{k} \Omega, \mathrm{Cp}_{\mathrm{p}}=0.37 \mathrm{nF}, \mathrm{R}_{\text {gap }}=75 \mathrm{k} \Omega$
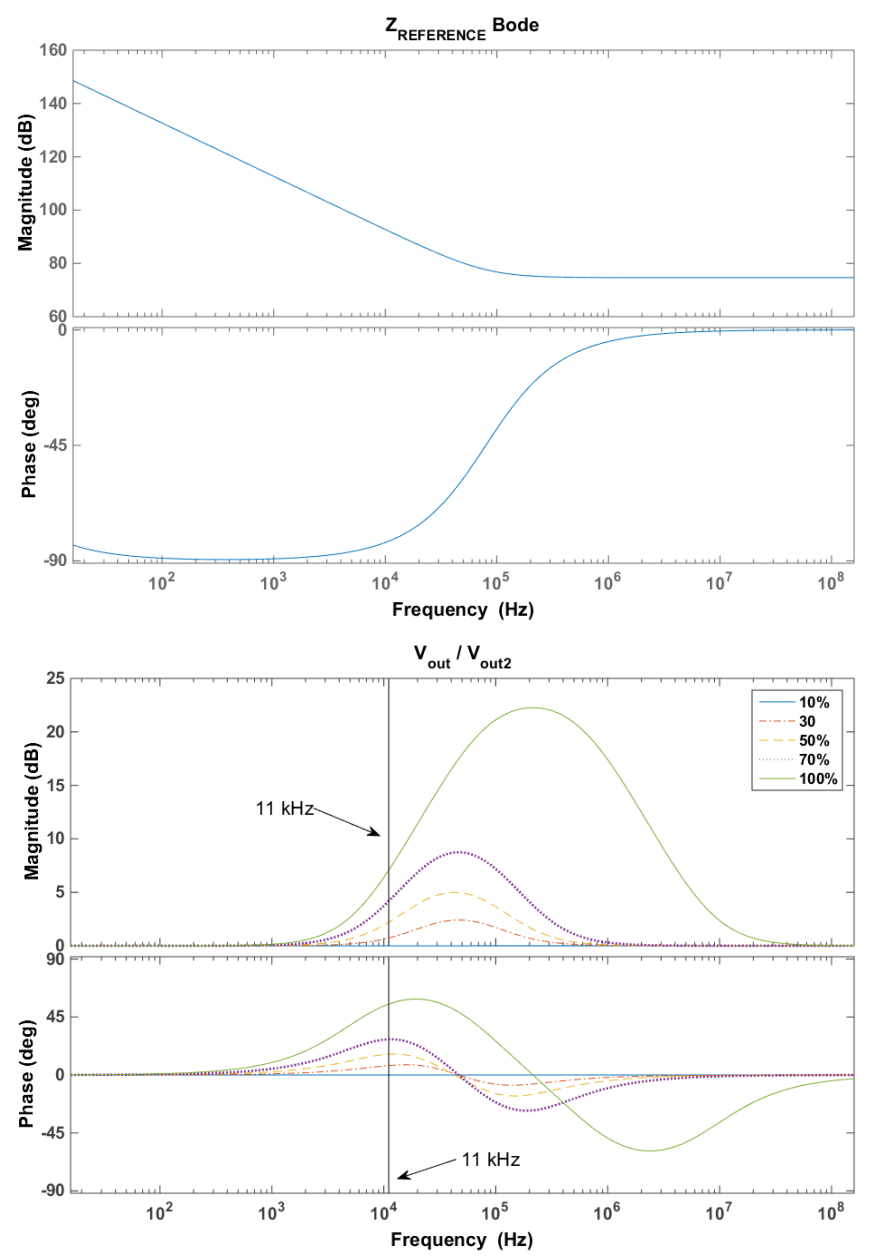

Fig. 3: Bode graphics for the magnitude and phase frequency response of the electrode\&cell-culture model in $[1,2] . Z_{\text {reference }}$ and $V_{\text {out }} / V_{\text {out } 2}$ in Fig. 1.

\section{OSCILLATION VOLTAGE (OV) IMPLEMENTATION}

Mathematically, the characteristic equation of the system in Fig. 1 would be:

$$
1+N(a) H(s)=0
$$

Where, $\mathrm{N}(\mathrm{a})=2 \mathrm{~V}_{\text {ref }} / \pi \mathrm{a}$ is the comparator describingfunction [12], and $\mathrm{H}(\mathrm{s})$ is the transfer function of the nonlinear feedback loop system: $\mathrm{H}(\mathrm{s})=\mathrm{H}_{\mathrm{BP}}(\mathrm{s}) \cdot \mathrm{H}_{\text {Electrode+Cell- }}$ Culture(s). Being $\mathrm{H}_{\mathrm{BP}}(\mathrm{s})$ and $\mathrm{H}_{\text {Electrode+Cell-Culture }}(\mathrm{s})$, respectively, the BP transfer function and the transfer function depicted in Fig 3, where, all the parameters are directly related to the electrode size, technology and biological cell-culture. 
To force oscillations, a pair of complex poles of the overall system has to be placed on the imaginary axis. The way to determine the oscillation conditions (gain, frequency and amplitude) is solving equation (1). This is equivalent to find the solution of this equation set $1+\mathrm{N}(\operatorname{aosc}) \cdot \mathrm{H}(\mathrm{s})=$ $\mathrm{s} 2+\mathrm{w} 2 \mathrm{osc})(\ldots)=0$.

There exists an oscillatory solution given in a graphical way in Fig. 4. Without loss of generality, it can be affirmed that the actual oscillation result (Fig.3) will be closed to this first approximation. These main oscillation parameters in Vout2, are function of ff.
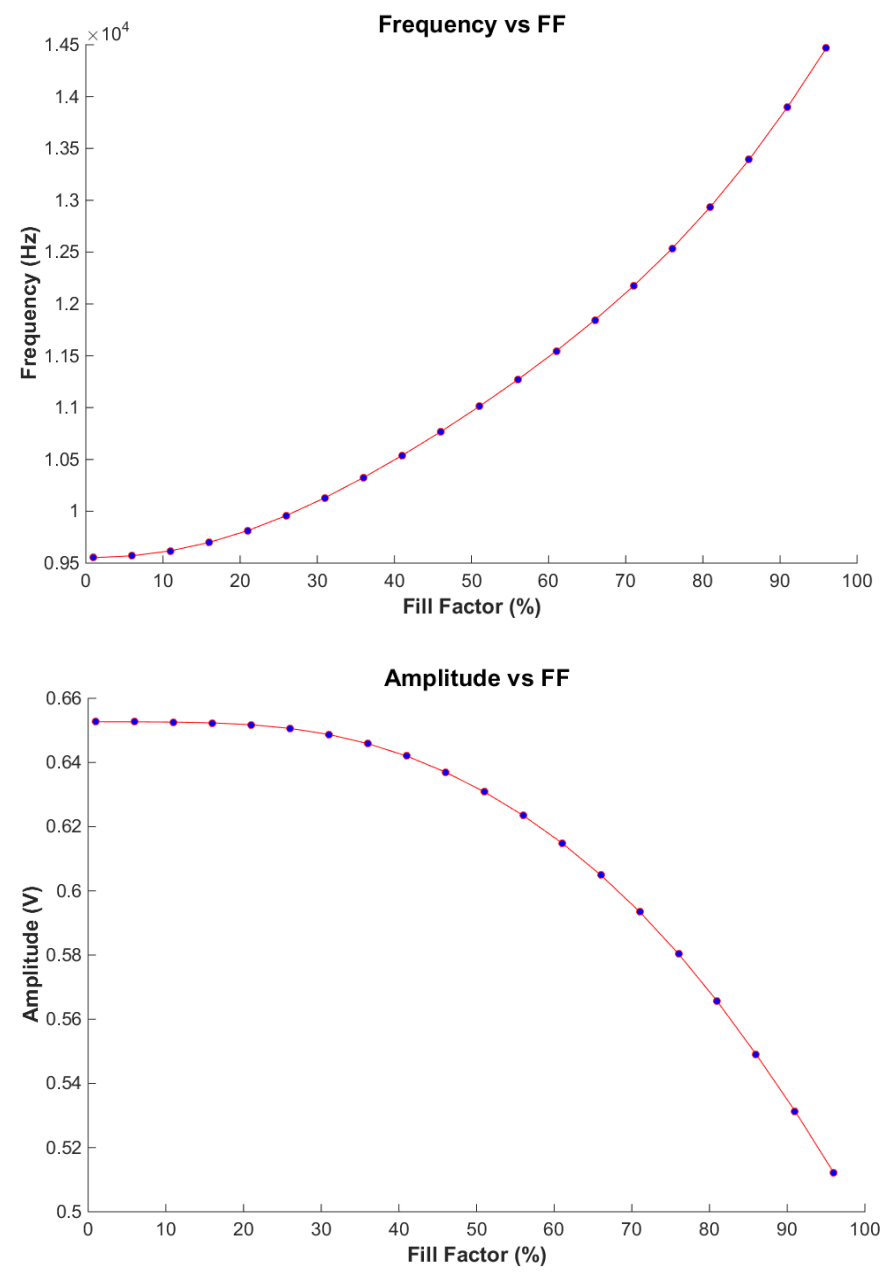

Fig. 4: Amplitude and frequency values for oscillations.

The BPF quality factor, Q, regulates the closed-loop system oscillatory behaviour in Fig. 1. A trade-off between obtaining a well-defined oscillation output and achieving enough margin in the oscillation frequency dynamic range is necessary. In this case, it is established that $\mathrm{Q}=1$, because a clear resonance peak is attained and, conversely, a suitable slope in the phase curve is also obtained which produces a significant change in the oscillation frequency for the different values of ff. The expected oscillation parameters, fosc and Aosc, are function of ff, as it is displayed in Fig. 4 for a microelectrode described in $[1,2]$.
These curves were obtained using matlab simulations. The fosc increases monotonically in the range $[9.5,14.5]$ $\mathrm{kHz}$, while the Aosc, increases, in the range of $[0.65,0.51]$ $\mathrm{V}$, when $\mathrm{ff}$ increases from 0 to $100 \%$. Due to the small voltage-level required at Vout, it can be considered the secondary output, Vout2, as the signal voltage output, thus improving the dynamic range. The obtained oscillation frequencies and amplitudes shown how there is a correlation with the ff increments. High ff values, convey into large oscillation amplitudes and frequencies, demonstrating the ff sensitivity of the proposed system. Fig. 5 (c) displays the FFT analysis of the output signal Vout2 for the studied cases of ff $(10 \%, 50 \%$ and $100 \%)$.
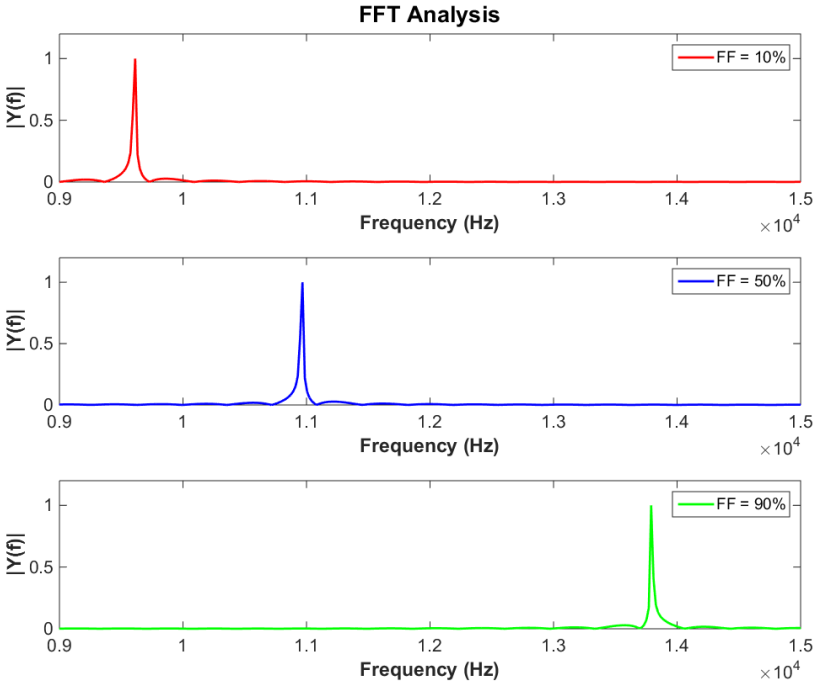

Fig. 5: FFT of the signal $V_{\text {out } 2}$ for 10,50 , and $90 \%$ fill-factor $(f f)$.

\section{PROCESSING UNIT AND DATA DISPLAY}

The digital section of the system prototype has three devices; a server installed in the engineering laboratory, a system-on-chip (SoC) powered device in the biomedical research laboratory and a data acquisition system inside the cell culture reactor which will drive the whole experiment. A bluetooth link is required between the $\mu \mathrm{P}$ and the SoC.

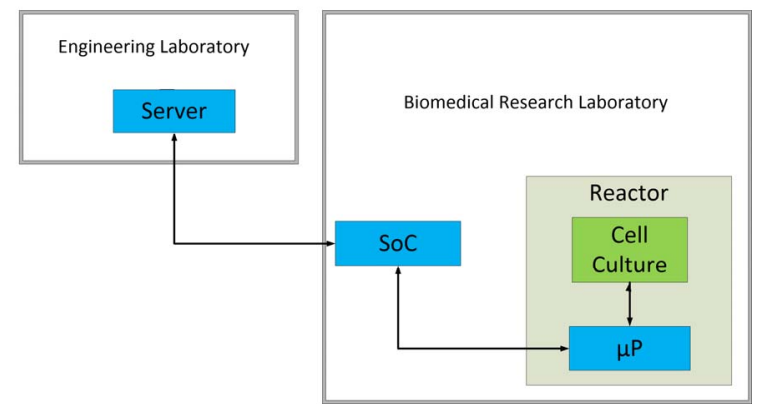

Fig. 6: Diagram for the system architecture.

The $\mu \mathrm{P}$ is an ARM Cortex-M4 device by STMicroelectronics. This device has a rich set of several peripheral devices. In our project we will use Analog to Digital Converter (ADC) for sampling data and Real-Time Clock (RTC) for synchronization. Cortex-M4 devices 
implements a Floating Point Unit (FPU) enabling them for executing signal processing algorithms.

The external device is designed using an Intel Edison platform. This SoC provides a Linux core with bluetooth and Wi-Fi links. The system communicates with the remote server which will act as a storage system and will serve data in a comprehensive manner using a web application directly implemented on it. The web application can define and modify experiment control variables like sampling time. This interface also shows real time data being gathered from the experiment. The interface is live on http://mixcell.dte.us.es/. A snapshot from the website showing data obtained from simulation models is depicted on Fig. 7.

This whole architecture designed is optimized for data integrity and power consumption. The $\mu \mathrm{P}$ system inside the cell cultures reactor is in standby mode for most of the time. When the RTC reactivates the whole system a connection between $\mu \mathrm{P}$ and external SoC is established using the bluetooth link and data is acquired. The $\mu \mathrm{P}$ then samples the amplitude value and calculates the frequency by the means provided by a Fast Fourier Transform (FFT). The device sends the calculated value to the external SoC which will decide, according to the experiment parameters stored on the database, which will be the next step for the experiment; take another measurement, measure another channel, read additional sensors data or send the system to standby mode for a while.
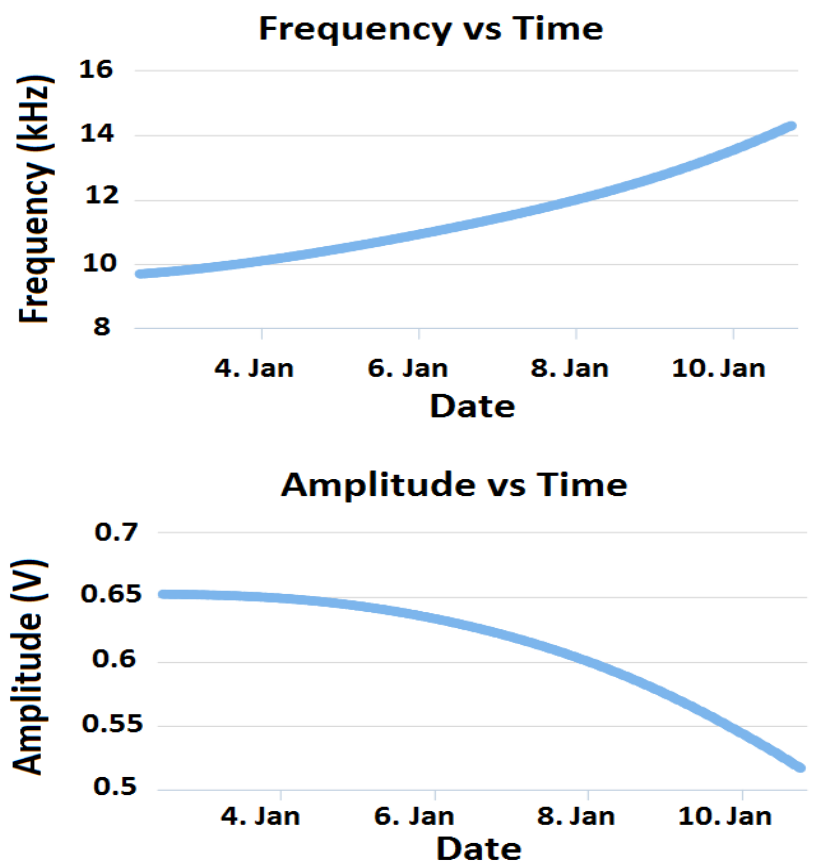

Fig. 7: Snapshot from the web application data displaying feature.

All data recovered by the SoC is stored both, locally and in the remote database in the server. An important remark from this architecture is that it can be implemented to support further functionality (for instance; multiple experiments being carried out by the biomedical researcher at the same time using multiple digital acquisition systems inside the reactor). Further improvement would include notifications based on researcher experiment definitions and program different alarms according to different experiment status.

\section{CONCLUSIONS}

It is presented a system for real-time monitoring cellculture assays with the proposed voltage oscillation techniques. The system allows to obtain oscillation signals with voltage amplitudes and frequencies correlated with the number of cell-culture (ff) in the assays. Baseline calibration is employed to derive the sensor sensitivities curves. The full system incorporates digital and wireless communication circuits for real time monitoring from a web application.

\section{ACKNOWLEDGMENT}

This work was supported in part by the Spanish funded Project: TEC 2013-46242-C3-1-P: Integrated Microsystems for Cell Culture Assays, co-financed with FEDER.

\section{REFERENCES}

[1] D. A. Borkholder. Cell-Based Biosensors Using Microelectrodes. PhD Thesis, Stanford University. 1998.

[2] X. Huang et al., "Simulation of Microelectrode Impedance Changes Due to Cell Growth", IEEE Sensors J., Vol. 4, No. 5, pp. 576-583, 2004.

[3] S. Grimnes, S. and O. Martinsen, Bio-impedance and Bioelectricity Basics. Second edition. Academic Press, Elsevier, 2008.

[4] I. Giaever and C. Keese, "Use of electric fields to monitor the dynamical aspect of cell behaviour in tissue culture" IEEE TBE, Vol. 33, No. 2. pp. 242-247, Feb 1986.

[5] I. Giaever \& C. R. Keese, "Micromotion of mammalian cells measured electrically", Proc. Nail. Acad. Sci. USA. Cell Biology, Vol. 88, pp. 7896-7900, Sep. 1991.

[6] J.O. Bockris \& A.K.N. Reddy, Modern Electrochemistry, Plenum Press, New York, 1970.

[7] A. Yúfera et al., "A Tissue Impedance Measurement Chip for Myocardial Ischemia Detection" IEEE TCASI, Vol. 52, No. 12, pp. 2620-2628, 2005

[8] P. Daza, A. Olmo, D. Cañete and A. Yúfera, "Monitoring Living Cell Assays with Bio-Impedance Sensors", Sensors and Actuators, Vol. 176, pp. 605-610, 2013.

[9] S. Radke et al., "Design and Fabrication of a Microimpedance Biosensor for Bacterial Detection", IEEE Sensor J., Vol 4, No. 4, pp. 434-440, 2004.

[10] G. Huertas, A. Maldonado, A. Yúfera, A. Rueda, J. L. Huertas, "The Bio-Oscillator: A Circuit for Cell-Culture Assays", IEEE TCASII, Vol. 62, pp. 164-168, 2014.

[11] A. Manickam, et al. "A CMOS Electrochemical Impedance Spectroscopy (EIS) Biosensor Array", IEEE BioCAS, Vol. 4, No. 6, pp. 379-390, 2010.

[12] A. Yúfera and A. Rueda, "Design of a CMOS closed-loop system with applications to bio-impedance measurements", Microelectronics Journal, Vol. 41, No. 4, pp. 231-239, 2010.

[13] G. Huertas et al., Oscillation-Based Test in Mixed-Signal Circuits, Frontiers in Electronic Testing. Springer. 2006.

[14] P. Fleischer et al, "A Switched Capacitor Oscillator with Precision Amplitude Control and Guaranteed Start-Up”, JSSC, Vol. 20, No. 2, pp. 641-647, 1985.

[15] Sohmyung H., et al, "Integrated Circuits and Electrode Interfaces for Noninvasive Physiological Monitoring”, IEEE T-BioCAS, vol. 61, n. 5, pp. 1522-1537, May 2014. 